\title{
PAPER \\ Design Requirements for Improving QoE of Web Service Using Time-Fillers
}

Sumaru NIIDA $^{\dagger, \dagger \dagger a)}$, Satoshi UEMURA ${ }^{\dagger}$, Members, and Etsuko T. HARADA ${ }^{\dagger \dagger}$, Nonmember

SUMMARY As mobile multimedia services expand, user behavior will become more diverse and the control of service quality from the user's perspective will become more important in service design. The quality of the network is one of the critical factors determining mobile service quality. However, this has mainly been evaluated in objective physical terms, such as delay reduction and bandwidth expansion. It is less common to use a human-centered design viewpoint when improving network performance. In this paper, we discuss ways to improve the quality of web services using time-fillers that actively address the human factors to improve the subjective quality of a mobile network. A field experiment was conducted, using a prototype. The results of the field experiment show that time-fillers can significantly decrease user dissatisfaction with waiting, but that this effect is strongly influenced by user preferences concerning content. Based on these results, we discuss the design requirements for effective use of time-fillers. key words: service quality, user experience, human-centered design, waiting, time-fillers, field study

\section{Introduction}

The market for mobile services now has billions of users. The recent expansion in high technology terminals and networks, such as smartphones, tablet computers and the thirdand fourth- generation mobile networks (IMT-2000 [1], IMT-Advanced [2]), provide wireless broadband communication services that have changed people's lifestyles. People can enjoy multimedia services on a cellular phone unconstrained by time and place. This trend will continue with the introduction of new mobile terminals and systems [3] and has led to massive diversification in the use of information. The rapid change in mobile use has brought into play factors that will affect service quality from the user-experience perspective. To gain information that helps mobile services to provide better quality, communication services need to be analyzed and evaluated from the user perspective, taking user attitudes and behavior into consideration.

One approach to analyzing service quality from the user perspective is called QoE (Quality of Experience) assessment, which has been discussed in association with the international standardization activities of the ITU (International Telecommunication Union). In ITU-T Appendix I to P.10/G.100 [4], QoE is defined as "The overall acceptability of an application or service, as perceived subjectively by the

\footnotetext{
Manuscript received October 29, 2012.

Manuscript revised March 21, 2013.

†The authors are with KDDI R\&D Laboratories Inc., Fujimino-shi, 356-8502 Japan.

${ }^{\dagger}$ The authors are with University of Tsukuba, Tsukuba-shi, 305-8577 Japan.

a)E-mail: niida@kddilabs.jp

DOI: 10.1587/transcom.E96.B.2069
}

end-user.

NOTES

a) Quality of Experience includes the complete end-toend system effects (client, terminal, network, services infrastructure, etc.).

b) Overall acceptability may be influenced by user expectations and context."

Based on this definition, quality should be evaluated subjectively in the situation actually experienced by users. Evaluations based on QoE are undoubtedly useful for offering users an optimal experience.

As described above, the control of service quality from the QoE perspective is becoming one of the primary emphases in mobile service design. There are many factors that affect mobile service quality, and the quality of the network is a critical factor because most mobile terminals are now connected to networks. However, the specification of criteria for network planning and operations has so far been based on network QoS (Quality of Service), such as network delay, jitter, packet loss, and bandwidth. In this physical perspective, improvements in service quality represent improvements in the QoS of physical networks. This is generally costly because of the vast size and complexity of leading-edge networks. A more cost-effective approach to finding the optimum trade-off between quality and cost is required, and a QoE approach can be part of the solution.

Typically, users attribute waiting time to a shortcoming in the QoS of an IP network. We have studied user tolerance for waiting time considering three factors: application classification, place of use and degree of relaxation [5]. These evaluation results are helpful as guidance for network planning. However, they do not provide information that helps to reduce dissatisfaction by some engineering strategy. A typical cognitive strategy among users is to deal with waiting time by multitasking. People reduce dissatisfaction with waiting time by taking their attention away from the waiting process. However, no service design method for making effective use of this strategy has really been investigated.

This paper discusses the design requirements for the use of time-fillers in web services. A time-filler is content that automatically appears on the screen of a terminal during waiting time. This may provide an alternative approach to the purely physical one of reducing waiting time by improving the network, in that it uses computer-human interactions to improve the QoE. The main contribution of this paper is to provide feedback for service design requirements based 
on a field experiment that used a prototype of the time-filler delivery method.

This paper is organized as follows. In Sect. 2, related works conducted to assess QoE of mobile services are summarized. In Sect. 3, service design utilizing time-fillers and an evaluation using prototyping is presented. In Sect. 4, the results of the field experiment are presented. In Sect.5, the design requirements are proposed. Finally, Sect. 6 concludes the discussion.

\section{Related Works}

Based on the definition of QoE, service quality should be evaluated subjectively under the conditions of real usage. Subjective quality assessment has a long history in the area of international standardization activities. Here we present related research on the subjective assessment of service quality, dividing the services into four traffic classes: conversational, interactive, streaming, and background as defined by Recommendation ITU-R M.1079 [6].

The typical use of the conversational class is telephony, including VoIP and videoconferencing. Subjective quality assessment has a long history in this class. ITU-T Recommendation P.800 [7] and its related documents define the methods for subjective assessment of voice quality. Recommendations ITU-R BT.500 [8] and BS.1116 [9] define subjective assessment of the quality of television pictures and audio systems, respectively. ITU also provides the methodology for deriving a subjective evaluation value from objective data.

In comparison with the interactive and streaming classes, the interactive class consisting of web browsing and downloading has a short history of subjective assessment. In web access, empirical rules such as the eight-second rule have been referred to in website design. ITU-T Recommendation G.1030 [10] defines the procedure for subjective quality assessment of website access on PCs and presents experimental results from subjective assessments of different types of users. Some research has also been conducted on QoE for web access services on PCs [11], [12] and downloading services [13], [14]. Some of the studies considered parameters other than the network QoS, such as price [15], [16] and usability [17]. There are very few studies that address mobile services in this class.

With regard to background class, it is difficult to perform subjective quality assessments of user experience since communication takes place under conditions such that users are not aware of the processes involved. Uemura et al. [18] propose a service control system that takes background service into consideration in multitasking situations.

There has also been some research on waiting time from a psychological perspective, which considered the duration of time as perceived by a human (termed "psychological time") in contrast to the absolute time measured by a clock. Relevant here is that the mental process involved in how individuals perceive the duration of time is closely related to the analysis of the effect of time-fillers. Peo- ple feel that a long time has passed when they are in a hurry, and become irritated as a consequence. Several time perception models have clarified features of the cognitive process involved in the over- or under-estimation of absolute time [19]-[21]. Some studies have aimed at reducing the dissatisfaction associated with waiting by using timefillers. Harrison et al. discussed how the visual design of the progress bar affects users' perception of time passing [22]. However, they did not compare the progress bar with other types of time-fillers. Antonides et al. [23] and Municho and Rafaeli [24] analyzed field experiments on the effect of various time-fillers in telephone queues (fillers include music, apologies, and information about location in the queues) based on the results of field experiments, and reported that such fillers can reduce dissatisfaction in waiting. However, it only discussed the sound-type fillers in telephone service. The effect of visual-type fillers was not evaluated. In addition to that, no reports have appeared demonstrating the design required for network systems and/or services to reduce the dissatisfaction elicited by waiting time from the user's perspective, considering psychological time.

\section{Field Study Method}

\subsection{Service Design for Time-Filler}

The authors have proposed a time-filler delivery system [25]. There are many kinds of time-filler in ICT terminals. Typical time-fillers are the status indicator type, such as a loading bar, a progress bar, and other short movies, which have already been implemented in many commercial mobile terminals. These prearranged time-fillers are stored in terminals by default, and the terminal plays them while waiting. Additionally, more flexible content such as news, trivia and advertisements can also be utilized as time-fillers. Such a media-type is expected to have a greater effect than an indicator type because it may attract more complete attention. However, this kind of context-dependent content needs updating periodically, requiring reconfiguration via the network. The delivery of media-type time-fillers should have little effect on system efficiency, which is especially important in mobile systems that do not have ample network resources.

One possible technology for time-filler delivery is multicast technology, which can achieve high efficiency because multiple users receive the same signal simultaneously. Some web services have already used this technology to push news content in the background [26]. It is also suitable to deliver other content, such as advertisements. Another possibility is to leverage off-hours. System utilization fluctuates with human activity, resulting in off-hours and rush hours. Therefore, the content delivery system can push content without affecting system performance by delivering it in off-hours.

We prototyped e-mail service over cellular phones by web script as an example of web service because it is a typical application that people use repeatedly in their daily lives. Trivia in text format were used as time-fillers because they 


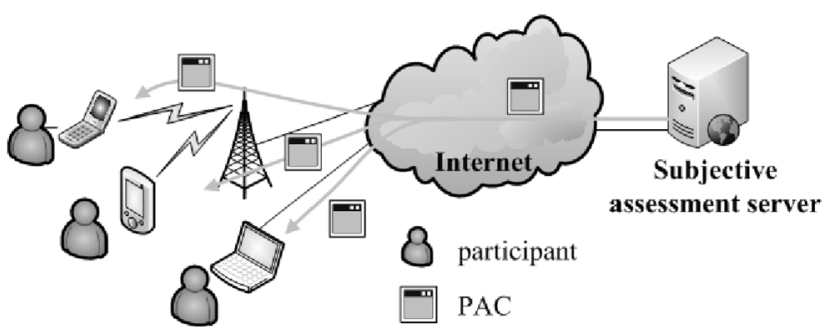

Fig. 1 Overview of the web script based system.

are appropriate content for time-fillers. They do not require a wideband network for delivery and may attract significant user attention.

\subsection{Investigating Waiting Time}

Conventional subjective assessments are conducted in a laboratory in order to obtain reliable results by controlling the experimental conditions. However, there are concerns about the applicability of such research findings because of the difference in conditions between a laboratory and real-world use. An experimental method is required that both controls the conditions and provides appropriate similarity to the actual usage situation ("ecological validity"). We developed an assessment system for field experiments that examined waiting time utilizing web scripts [27]. An overview of the system is depicted in Fig. 1. This system consists of a subjective assessment server (SAS) and user terminals. The SAS distributes web script content, which we call PAC (Program for Artificial Context), that contains experimental tasks and receives responses to the tasks from participants over a network. This system can control the waiting time duration regardless of the network conditions by controlling the display transition in the web script. It also allows experiments to be conducted with ecological validity because participants can use their own cellular phone and execute the content at an arbitrary time and place. Participants can conduct the field evaluation anywhere, since the PAC can be run without a connection to a network. Because of these features, this evaluation system is suitable for clarifying the mental processes related to waiting in an actual situation.

Once the participants have obtained the PAC, all necessary procedures can be accomplished according to the instructions for the PAC. The mobile service can be simulated by a displayed picture because users recognize their situation only through the events on the display. Our proposed system can control the waiting-time duration regardless of network conditions by controlling the display transitions with a web script. It can also conduct experiments with ecological validity because participants use their own cellular phone, since most commercial mobile terminals support Flash Lite by default. They can execute the content at arbitrary times and places of their choice, as with any ordinary use. Because of these features, this evaluation system is suitable for investigating the subjective quality of networkrelated waiting in actual situations.

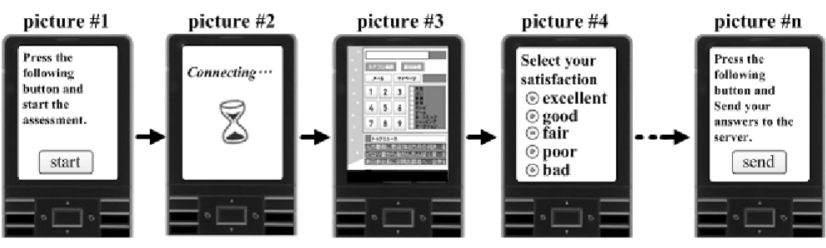

Fig. 2 Example of a PAC.

For example, assume the situation of accessing a website using a cellular phone, the PAC can be configured to assess QoE for waiting time for web access as shown in Fig. 2. In Fig. 2, as soon as the start button is pressed, the displayed picture is turned into an hourglass animation. The hourglass animation is displayed for several seconds, and then the top page of the website is displayed. Note that a picture appropriate for the purpose of the assessment can be used. Next, the questionnaire on satisfaction regarding waiting time is displayed. Finally, answers for several questionnaires are sent to the SAS by pressing the send button. The timing of replacing pictures associated with specific key events can also be controlled.

\subsection{Evaluation Method}

The test was conducted with the assessment system described above. In this experiment, the program emulated the sending of an e-mail with an attachment by a cellular phone, which began with pressing the send button and finished with the display of the sign indicating that the e-mail transmission was completed. During the waiting time, one of five types of time-fillers was displayed on the screen of the cellular phone: loading bar (LB), discrete progress bar (PB), continuous $\mathrm{PB}$, loading bar with trivia, and continuous progress bar with trivia. The discrete $\mathrm{PB}$ and the continuous PB showed progress respectively at each $25 \%$ and $1 \%$ mark of the total time to send the e-mail. Figure 3 shows screens displaying the LB, PB and the PB with trivia. There are twelve types of trivia, each consisting of around 150 words in Japanese. These were chosen from trivia books arbitrarily by the authors. For instance "the reason for calling the last runner in a relay an anchor", "the sex of a sea turtle is determined by the temperature of the ground at egg laying".

The number of participants in the experiment was 480 . The participants were all residents of Japan and registered with an online survey company. A stratified sampling by sex and age group was used in the experiments, divided into 15-19, $20 \mathrm{~s}, 30 \mathrm{~s}, 40 \mathrm{~s}$, and $50 \mathrm{~s}$. A repeated-measures design was used, with each participant being tested under five content blocks. There were five waiting-time conditions inside each block of $4,6,8,10$, and 12 seconds. The order of the blocks and waiting-time durations inside blocks was randomly assigned for each participant. Participants evaluated their satisfaction with the waiting-time duration on a five-point scale $(5=$ very satisfied, $4=$ satisfied, $3=$ neither satisfied nor dissatisfied, 2 = dissatisfied, 1 = very dissatisfied). After the evaluation of twenty five waiting processes, 


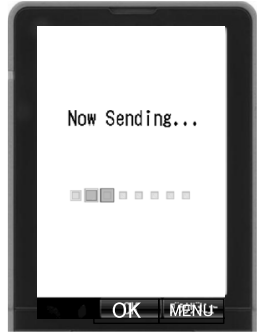

(a) Loading Bar (LB)

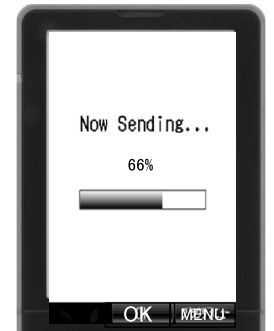

(b) Continuous Progress Bar (PB)

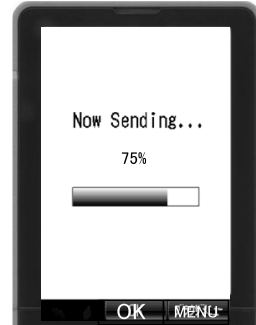

(c) Discrete PB (each 25\% mark)

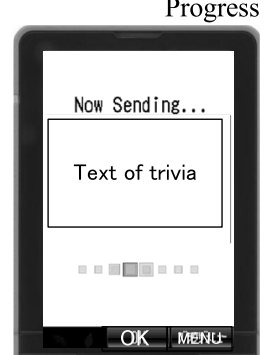

(d) LB with Trivia

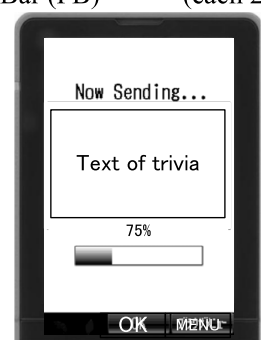
with Trivia (e) Continuous PB

Fig. 3 Screen display with time-fillers.

participants were asked their sex, age, preferences for trivia and other demographic questions, and required to send the responses to the server over the network.

\section{Results}

At first, the effect of demographic parameters, sex and age, was confirmed by a four-way analysis of variance with waiting time and filler type as repeated factors, sex and age as grouping factors. The results showed that there are not significant differences between sex and age groups at the 0.05 level. Hereafter, sex and age are not considered in evaluation.

\subsection{Effect of Time-Fillers}

Figure 4 shows the average participant rating for each timefiller on a five-point scale (MOS: Mean Opinion Score). A two-way analysis of variance was conducted with the filler type as the grouping factor and the waiting time as the repeated factor. The main effect of the waiting time duration, $F(4,7664)=890.1, p<.001$, was significant, with the MOS significantly declining as the waiting time grew longer. The main effect of the filler type, $F(4,7664)=$ 49.7, $p<.001$, and the interaction between the waiting time and the filler type, $F(16,7664)=17.8, p<.001$, were also significant. This means that the differences in MOS between filler types are statistically significant at particular waiting-time durations. The results of the Fisher's LSD multiple comparison test were as follows: the continuous $\mathrm{PB}$ with trivia produced significantly greater satisfaction than the others at 8-10 seconds at the 5\% level; there were nonsignificant differences between LB and discrete PB at 6-12 seconds; the continuous PB showed the same effectiveness

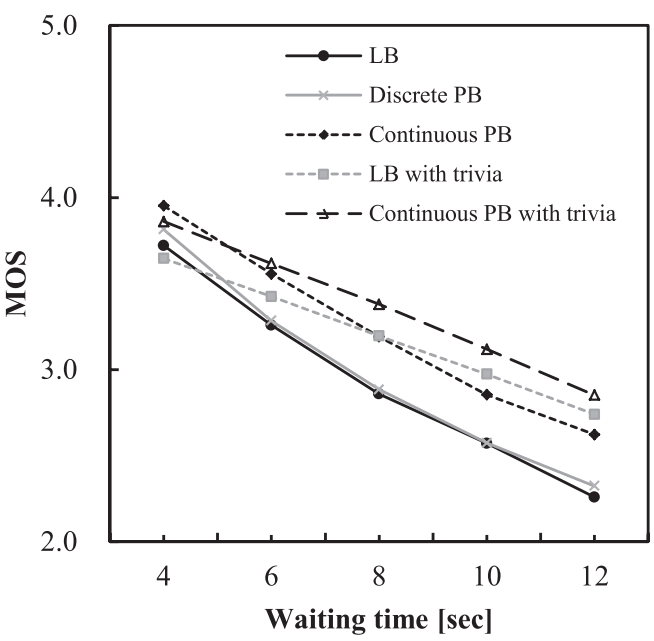

Fig. 4 Effect of time-fillers on MOS.

as the continuous PB with trivia at 4-6 seconds but less than the LB with trivia at 10-12 seconds.

These results showed that the use of either a continuous $\mathrm{PB}$ or trivia can significantly reduce dissatisfaction with waiting. However, the continuous PB may work differently than trivia. The continuous PB showed a constant effect regardless of the waiting-time duration. Using trivia can have an additional effect on PB but only in the longer waiting times. If the waiting time is much shorter than the required reading time, there is the potential for a negative impact on satisfaction. This is attributed to the fact that trivia require extra time to read. In this experiment, each trivia item contained around 150 letters, requiring several seconds to read through. Thus, while trivia can be very effective, there is also a risk of adverse effects.

\subsection{Effect of Preference for Time-Fillers}

The previous results showed that the effect of trivia depends on the waiting-time duration. In this section, the effect of trivia is analyzed based on user responses to a questionnaire on preference for trivia. Participants were asked to rate their preferences for trivia on a five-point scale $(5=$ very interesting, $4=$ interesting, $3=$ neither interesting nor uninteresting, $2=$ uninteresting, $1=$ very uninteresting). The number of responses at each level was 47, 204, 183, 33, and 6, respectively. Seven participants who did not answer this question were excluded from the analysis. We merged positive levels (very interesting and interesting) and negative levels (uninteresting and very uninteresting) to make three groups.

Figure 5 shows the MOS versus waiting time for participants grouped together by their preference for trivia. A two-way analysis of variance was conducted on data of the continuous PB with trivia. The results yielded a main effect for the waiting time that was significant, $F(4,1880)=$ $92.1, p<.001$. The main effect of preference, $F(2,470)$ $=25.8, p<.001$, was significant, however, their interaction was non-significant, $F(8,1880)=1.37, p=.206$. A detailed analysis by the Fisher's LSD multiple comparison 


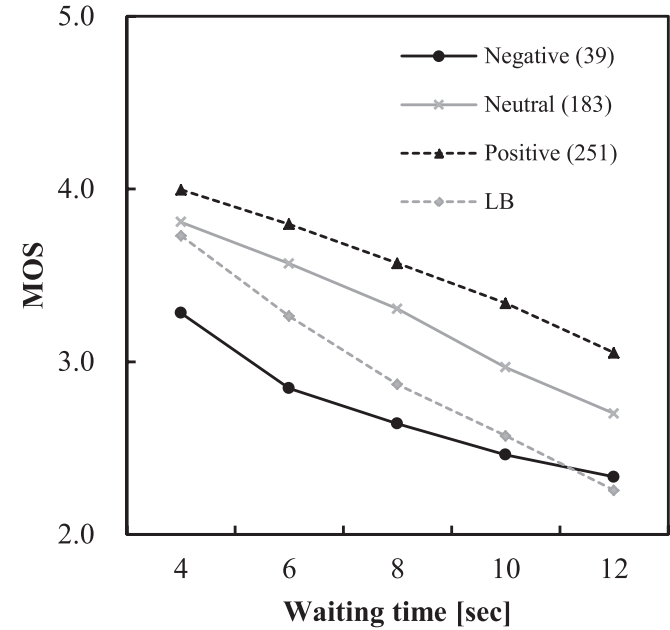

Fig. 5 Effect of preference for trivia on MOS (continuous progress bar with trivia).

test showed that there were significant differences between all combinations of groups regardless of the waiting time at the 5\% level. As shown in Fig. 5, the MOS of the negative group was lower than that for LB. This result can be interpreted two ways. First, it suggests that this index actually represented the participants' preference for trivia. Second, this index indicated how highly the participants rated satisfaction and preference as individual traits. To examine the effect of preference, we conducted the same analysis on the result of continuous PB without trivia as shown in Fig. 6. A two-way analysis of variance yielded a main effect for the waiting time that was significant, $F(4,1880)=187.6$, $p<.001$. However, the main effect of preference, $F(2,470)$ $=2.21, p=.110$, and their interaction, $F(8,1880)=1.77$, $p=.078$, were non-significant. The difference of MOS between preference groups was significant only if the trivia were displayed. This result shows that the effect of trivia is highly dependent on user preferences. In this experiment, more than half of the participants had positive feelings for trivia, which contributed to the noteworthy ability of trivia to increase the mean opinion score. If some unpopular content were utilized as the time-filler, opinion scores would deteriorate significantly.

\subsection{Behavior Analysis}

In practice, the participants in the negative group refused to even read trivia. Figure 7 shows the residence time in content, which is the time interval between pushing the button to start sending e-mail and moving on to the evaluation stage after finishing sending e-mail or reading trivia. The residence time was generally longer than the set waiting time because it included a response time to allow for pushing buttons and reading trivia. A two-way analysis of variance yielded a main effect for the waiting time, $F(4,1880)=64.0$, $p<.001$, and the preference, $F(2,470)=11.9, p<.001$, which were significant. The interaction effect was also significant, $F(8,1880)=1.97, p=.047$. This means that the

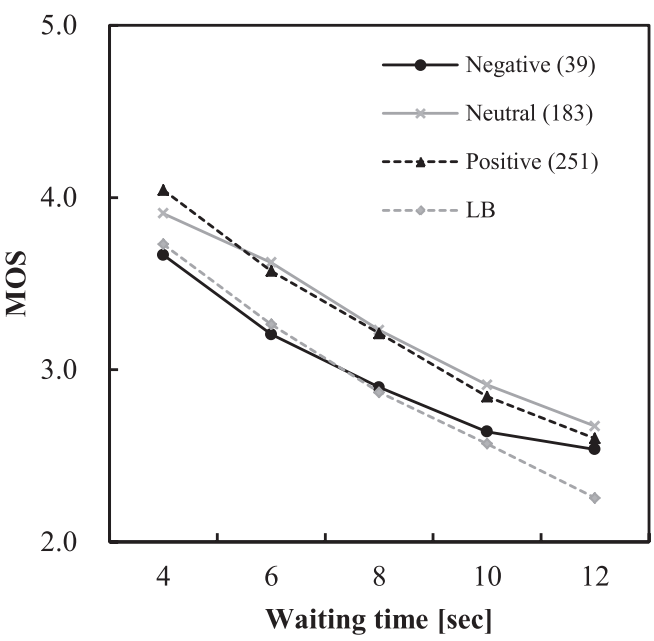

Fig. 6 Effect of preference for trivia on MOS (continuous progress bar without trivia).

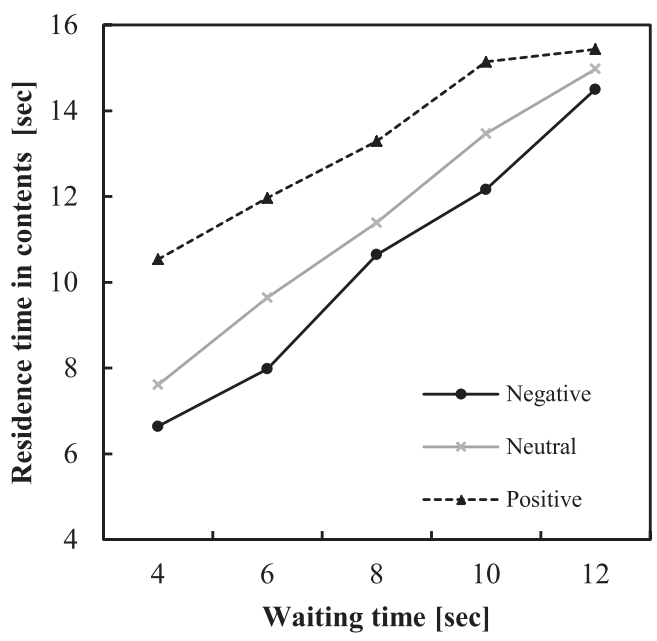

Fig. 7 Voluntary residence time in content.

differences in residence time between filler types were statistically significant, in particular waiting time length. The results of a simple main effect test indicated that the average time interval for the negative and the neutral groups was significantly shorter than that of the positive group with 4-10 seconds waiting time. With 12 seconds of waiting time, the difference between groups disappeared.

Figure 8 shows the MOS versus waiting time for participants grouped together by their status on reading trivia. As shown in this figure, the MOS of the participants who did not read the trivia was lower than that for the participants who read through or read until the e-mail transmission was complete. This result also indicates that the effect of trivia is dependent on the status if participants finish reading them. Table 1 shows a cross tabulation of the number of participants by MOS and reading status. This also indicated that most of the participants who had negative feelings for trivia did not even read them. This may indicate that the strong dissatisfaction associated with waiting time caused the dis- 


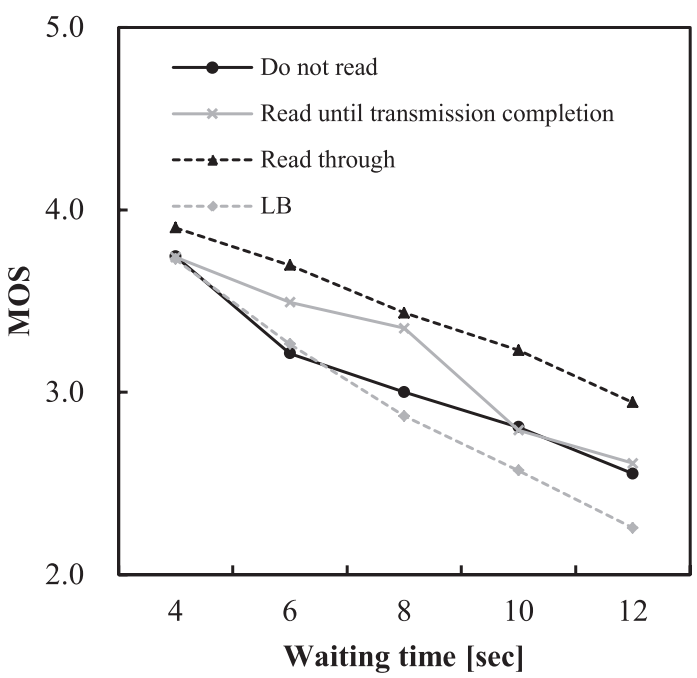

Fig. 8 Effect of reading condition on MOS (continuous progress bar with trivia).

Table 1 Cross tab displaying the number of participants by MOS and reading status.

\begin{tabular}{lcccc}
\hline MOS & 5 & 4 & 3 & 2 \\
\hline $\begin{array}{l}\text { Do not read } \\
\begin{array}{l}\text { Read until } \\
\text { transmission completion }\end{array}\end{array}$ & 1 & 25 & 37 & 11 \\
\begin{tabular}{l} 
Read through \\
\hline
\end{tabular} & 45 & 172 & 110 & 11 \\
\hline
\end{tabular}

satisfaction with the trivia, and this was thought to explain the negative attitude that was shown towards them. From these results, the residence time can be used as an indicator of preference and/or reading status.

\section{Design Requirements for Time-Filler Delivery}

The results show that time-fillers strongly affect the reduction of dissatisfaction while waiting. Based on the results of the field study, we propose design requirements for a timefiller delivery system.

- The system should implement both indicator type and media-type time-fillers. Those time-fillers are more effective in combination than singly, because each addresses a different aspect of the problem.

- It is quite important to know whether or not users feel positive about the media-type fillers and their content, which may yield negative effects if presented to users who are uninterested.

- It is strongly recommended that systems be equipped with a function to estimate user preference for this service and/or content. The voluntary residence time in particular content can be used as a metric.

- If the system can predict a waiting time length, it is recommended that media-type fillers only be used when the waiting time will be long enough to enjoy the content. Media-type fillers may have a negative effect if they are presented in a time too short for their enjoyment.

One way to satisfy these requirements in a mobile system is described below. When a user pushes a button to obtain a large item from a server, the user's terminal can estimate the download time from the item's size and the expected download speed. The size is usually described in the first response message from the server in HTTP, POP or IMAP. The expected throughput rate is reported by a base station when a mobile terminal is allocated resources by a scheduler. After estimating the waiting time, the mobile terminal can decide which of the indicator types, or what combination of indicator and media-type, can be displayed by comparing the expected waiting time length with a baseline (5 seconds can be used). During the downloading, an appropriate time-filler is displayed in a sub-window. After finishing the download, the progress indicator transforms into a button to close the sub-window. The voluntary residence time can be estimated by measuring the time between pushing the button to start downloading and pushing the button that closes the sub-window.

\section{Conclusion}

In the past, network performance has been improved by improving the objective QoS. Such a physical approach is effective for most users but it increases the cost. Cost-effective approaches are required, and these can be realized by actively addressing the human factor. A delivery method that uses a time-filler to occupy the user during downloading is one way to combine user interface technology and network technology to improve perceived network performance. It is expected that a communications system will achieve a good balance between superior cost performance and a high degree of satisfaction by introducing a human-centered approach when developing new technologies.

In this paper, the authors evaluated the effect of different time-fillers on perceived mobile service quality. Field experiments showed that time-fillers can significantly reduce a user's dissatisfaction while waiting for the system to complete an action. The display of trivia has an additional effect beyond the effect of loading bars and progress bars, but this works differently for different users. It has a negative effect when users do not like the content. To use timefillers effectively, each terminal needs to select content that matches its user's preferences. Experimental results show that users who dislike the content skip it and proceed to the next action. It makes sense that the user's choice of residence time with the content can be used as an index of user preference for the time-filler.

In this study, the action of sending an e-mail with an attachment was selected as an example of ICT service. These results may be broadly applicable to waiting situations in ICT usage, such as receiving e-mails with attachments and downloading various contents. The results may also extend to traffic control during a multitasking operation on a smart- 
phone. These results indicate that users who turn their attention to a foreground task become less concerned about the background task, so that reduced throughput on a background task may not cause reduced satisfaction.

In this study, text was used as the time-filler content. If content is used in this system that demands more attention than text, such as video, it is highly likely that the size of the effect will change. However, the qualitative effect of the system may be the same.

\section{References}

[1] Recommendation ITU-R M.1457-8, "Detailed specifications of the radio interfaces of International Mobile Telecommunications-2000 (IMT-2000)," May 2009.

[2] Recommendation ITU-R M.1822, "Framework for services supported by IMT," 2007.

[3] Report ITU-R M.2072, "World mobile telecommunication market forecast," 2006.

[4] ITU-T Appendix I to P.10/G.100, "Definition of QoE," 2007.

[5] S. Niida, S. Uemura, and H. Nakamura, "Mobile services - User tolerance for waiting time," IEEE Vehicular Technology Magazine, vol.5, no.3, pp.61-67, Sept. 2010.

[6] Recommendation ITU-R M.1079-2, "Performance and quality of service requirements for IMT-2000 access networks," 2003.

[7] ITU-T Recommendation P.800, "Methods for subjective determination of transmission quality," 1996.

[8] Recommendation ITU-R BT.500-11, "Methodology for subjective assessment of the quality of television picture," 2002.

[9] ITU-T Recommendation BS.1116, "Methods for the subjective assessment of small impairments in audio systems including multichannel sound systems," 1997.

[10] ITU-T Recommendation G.1030, "Estimating end-to-end performance in IP networks for data applications," 2005

[11] A. Bouch, A. Kuchinsky, and N. Bhatti, "Quality is in the eye of the beholder: Meeting users' requirements for internet quality of service," Proc. CHI'00, pp.297-304, April 2000.

[12] E. Ibarrola, F. Liberal, I. Taboada, and R. Ortega, "Web QoE evaluation in multi-agent networks: Validation of ITU-T G.1030," Proc. ICAS'09, pp.289-294, April 2009.

[13] K. Nomura, K. Yamori, E. Takahashi, T. Miyoshi, and Y. Tanaka, "Waiting time versus utility to download images," Proc. APSITT 2001, pp.128-132, Nov. 2001

[14] T. Kaji, Y. Minoda, K. Tsukamoto, and S. Komaki, "Radio agent realizing user utility-based resource allocation in wireless spot access," Proc. ISCIT 2004, pp.286-289, Oct. 2004.

[15] T. Okamoto and T. Hayashi, "Analysis of service provider's profit by modeling customer's willingness to pay for IP QoS," Proc. Globecom'02, vol.2, pp.1549-1553, Nov. 2002.

[16] Y. Liu, A.H. Ngu, and L.Z. Zeng, "QoS computation and policing in dynamic Web service selection," Proc. WWW 2004, pp.66-73, May 2004.

[17] P. Concejero, J. Patrocinio, and D. Merino, "Usability evaluation of mobile services,” Proc. ICIN'08, Oct. 2008.

[18] S. Uemura, S. Niida, and H. Nakamura, "Service control system based on behavioral characteristics of user multitasking," Proc. CENTRIC 2011, pp.22-27, Oct. 2011.

[19] R.E. Hicks, G.W. Miller, G. Gaes, and K. Bierman, "Concurrent processing demands and the experience of time passing," American Journal of Psychology, vol.90, pp.431-446.

[20] P. Fraisse, "Perception and estimation of time," Annual Review of Psychology, no.35, pp.1-36, 1984.

[21] R.A. Block and D. Zakay, "Models of psychological time revisited," in Time and Mind, ed. H. Helfrich, pp.171-195, Kirkland, 1996.

[22] C. Harrison, Z. Yeo, and S.E. Hudson, "Faster progress bars: Ma- nipulating perceived duration with visual augmentations," Proc. CHI 2010, pp.1545-1548, April 2010.

[23] G. Antonides, P.C. Verhoef, and M. van Aalst, "Consumer perception and evaluation of waiting time: A field experiment," J. Consumer Psychology, no.12, pp.193-202, 2002.

[24] N. Munichor and A. Rafaeli, "Numbers or apologies? Customer reactions to telephone waiting time fillers," J. Applied Psychology, no.92, pp.511-518, 2007.

[25] S. Niida, S. Uemura, H. Nakamura, and E. Harada, "Field study of a waiting-time filler delivery system," Proc. MobileHCI 2011, pp.177-180, Aug. 2011.

[26] Y. Cho, K. Kang, and H. Shin, "Seamless multimedia broadcasting over cdma2000 BCMCS networks,” Proc. ICC'07, pp.5628-5635, June 2007.

[27] S. Uemura, S. Niida, and H. Nakamura, "A Web script-based field evaluation method to assess subjective quality of mobile services," IEICE Trans. Commun., vol.E94-B, no.3, pp.639-648, March 2011.

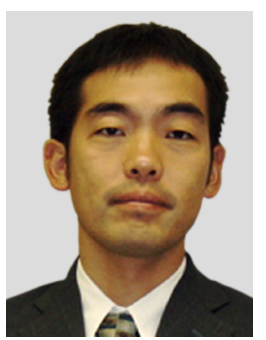

Sumaru Niida received B.E. and M.E degrees in electrical and computer engineering from Yokohama National University in 1994 and 1996, respectively. He joined the Research and Development Laboratories of Kokusai Denshin Denwa Company Ltd. (currently KDDI) in 1996, as a research engineer. His research interests include quality assessment and design of communication services. He is currently a research engineer in the Communications Network Planning Laboratory of KDDI R\&D Laboratories, Inc. He received the Young Researcher's Award from IEICE in 2002, and the Best Paper Award at the WWRF 24th Meeting in 2010.

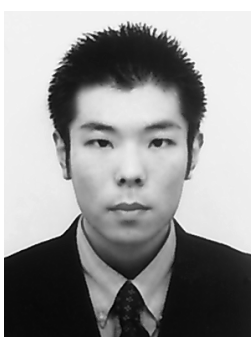

Satoshi Uemura received B.E., M.E. and Ph.D. degrees in electronics and information engineering from Hokkaido University, Japan in 2000, 2002 and 2005, respectively and in 2005 , he joined KDDI R\&D Laboratories Inc. His research interests include QoE assessment in mobile services and traffic control systems in communication broadcasting integrated services. $\mathrm{He}$ is currently a research engineer in the IP Communications Quality Laboratory of KDDI R\&D Laboratories Inc. He received the English Session Award from the TM committee of IEICE in 2007, the Young Researcher Award from the MoMuC committee of IEICE in 2008 and the Young Researcher's Award from IEICE in 2009.

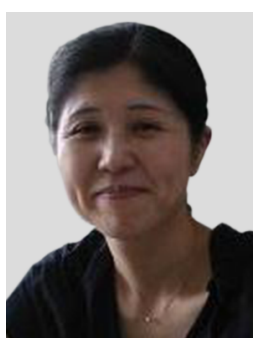

Etsuko T. Harada Ph.D. in psychology, joined IBM Tokyo Research Center in 1986 as a researcher in the Cognitive Engineering Group, after she finished her graduate degree in psychology at the University of Tsukuba. She started her studies on human-artifact interaction there, in addition to her original research on human memory, cognition, and learning. Since 1989, she has continued her research in both experimental cognitive psychology and cognitive engineering, first at Hosei university and then the University of Tsukuba. She has a wide range of interests in the field of human-artifact interactions, focusing especially on the relationship between cognitive-usability and aging in recent years. Currently she is a Professor at the University of Tsukuba, and is managing a JST-RISTEX project at the Center for Usability and Aging Research. 